\section{Selbstorganisation als Kern der Synthetischen Biologie}

Ein Beitrag zur „Prospektiven

Technikfolgenabschätzung“

von Jan C. Schmidt, Hochschule Darmstadt

Von der Synthetischen Biologie werden zentrale Beiträge zur Technologie der Zukunft erwartet. Was die Synthetische Biologie selbst jedoch kennzeichnet, ist offen. Die „Prospektive Technikfolgenabschätzung“ (ProTA) verfolgt hier das Ziel, in frühen Phasen wissenschafts- und techniknahe Reflexionen einzuleiten, begriffliche Klärungen herbeizuführen und Gestaltungsoptionen zu eröffnen. Der vorliegende Beitrag zeigt, dass das systemund strukturwissenschaftliche Konzept der Selbstorganisation für ein adäquates und gehaltvolles Verständnis der Synthetischen Biologie unerlässlich ist: Sie kann als Biotechnowissenschaft zur technischen Nutzbarmachung von Selbstorganisation(sprinzipien) angesehen werden. Hieran anschließend werden prinzipielle Grenzen der Konstruierund Kontrollierbarkeit von Biosystemen erörtert. Vor diesem Hintergrund kann ProTA zu einer realistischen Einschätzung der Potenziale und Realisierungsbedingungen der Synthetischen Biologie beitragen.

\section{Einleitung}

Die Synthetische Biologie wird als zentrale $\mathrm{Zu}-$ kunftstechnowissenschaft angesehen. Eine Herstellung künstlichen Lebens im Labor sei gelungen - zumindest der erste Schritt dahin, heißt es. Craig Venter trat mit seinem Team im Mai 2010 vor die Presse und verkündete den Durchbruch. Er präsentierte ein ,proof of concept“, mit anderen Worten: Synthetische Biologie sei möglich. Aus „vier Flaschen Chemikalien“ könne man „Leben erzeugen“. Das sei nicht nur wissenschaftlich interessant, sondern auch wirtschaftlich relevant. Eine ,neue industrielle Revolution" stehe vor der Tür, so Venter.

Schaut man genauer hin, so relativiert sich der Anspruch des Revolutionären. Venter hat lediglich ein modifiziertes Genom der Bakterienart „Mycoplasma mycoides “ synthetisiert, das dann in das Bakterium der Spezies „Mycoplasma ca- pricolum" eingeschleust wurde. Außer der eigenen Vermehrung, verbunden mit den dazu nötigen Stoffwechselprozessen, kann das Bakterium jedoch nichts Neues leisten.

Die Ankündigung rief Kritiker auf den Plan, gerade in Deutschland: Venter spiele Gott. Zwischen Entrüstung und Euphorie oszillierte fortan der öffentliche wie wissenschaftliche Diskurs. Damit jedoch wurden und werden Gestaltungsoptionen verspielt. Diese sind aber durchaus vorhanden; schließlich befindet sich die Synthetische Biologie in einem frühen Entwicklungsstadium. In diesem setzt das Konzept der Prospektiven Technikfolgenabschätzung (ProTA) an. ProTA ist eine antizipative Wissenschafts- oder Forschungsfolgenforschung (Liebert/Schmidt 2010). Sie basiert auf einer vierfachen Rahmenorientierung: Frühzeitigkeits-, Intentions- und Gestaltungsorientierung sowie einer Orientierung am wissenschaftlich-technischen Kern.

Im Folgenden wird keine umfassende ProTA durchgeführt; vielmehr soll beispielhaft nach dem wissenschaftlich-technischen Kern der Synthetischen Biologie gefragt werden. ${ }^{1}$

\section{Was ist Synthetische Biologie?}

Was sich hinter dem Kollektivsingular „Synthetische Biologie" verbirgt, ist alles andere als klar. Das mag zunächst nicht verwundern: Denn der Begriffskarriere nach ist die Synthetische Biologie eine junge Wissenschaft. Eric Kool prägte den Begriff auf dem Jahrestreffen der American Chemical Society im Jahr 2000. Doch genauer betrachtet findet sich der Begriff „Synthetische Biologie" schon vor 100 Jahren, auch wenn dieser seither kaum verwendet wurde.

Neue catch- oder buzzwords (wie „Synthetische Biologie") sind nicht harmlos - das weiß die TA. Begriffe legen fest, schließen ein oder aus, nähren Hoffnungen oder Befürchtungen, erschließen oder verschließen Forschungskorridore. Begriffsfestlegungen sind stets normativ. Sie bilden nicht nur die Grundlage für wissenschaftliches Arbeiten, sondern den Hintergrund für öffentliche Debatten und politische Entscheidungen. Drei unterschiedliche, durchaus umstrittene Begriffsfestlegungen, was „Synthetische Biologie" kennzeichnet, lassen sich finden: 
(1) das Ingenieursverständnis: Eine High-Level Expert Group der Europäischen Kommission hatte im Jahre 2005 eine Definition vorgelegt, die offenbar weithin Konsens ist: „Synthetic biology is the engineering of biology: the synthesis of complex, biologically based (or inspired) systems, which display functions that do not exist in nature. This engineering perspective may be applied at all levels of the hierarchy of biological structures - from individual molecules to whole cells, tissues and organisms. In essence, synthetic biology will enable the design of 'biological systems' in a rational and systematic way." (EC 2005, S. 5) Das entspricht dem, was die Akademie der Technikwissenschaften heraufziehen sieht, nämlich: Synthetische Biologie als „Geburt einer neuen Technikwissenschaft" (Pühler et al. 2011). Diese Verständnisweise basiert auf Abgrenzungen relativ zur Biologie, nicht zur Biotechnologie: Nicht Theorie (wie in der Biologie), sondern Technik (wie in den Technikwissenschaften) sei Ziel der Synthetischen Biologie. Damit wird ein Anspruch eines Epochenbruchs vertreten, nach dem Synthetische Biologie etwas kategorial Neues oder Anderes sei.

(2) das Künstlichkeitsverständnis: Dieses rekurriert weniger auf Ziele als auf Objekte. So wurde die Synthetische Biologie im Rahmen des EUTESSY-Projekts über neuartige Biotechno-Objekte definiert, nämlich über solche, ,that do not exist as such in nature" (TESSY-Consortium 2008). Vergleichbares findet sich in obiger berühmter Definition der EU-Expertengruppe (EC 2005, S. 5). Mit der Erschaffung von nichtnatürlichen Biosystemen habe es die Synthetische Biologie zu tun, so auch DFG, acatech und Leopoldina (DFG et al. 2009, S. 7): „Dabei können Eigenschaften entstehen, wie sie in natürlich vorkommenden Organismen bisher nicht bekannt sind." Synthetische Biologie sei damit gerade durch die Nicht-Natürlichkeit bzw. Künstlichkeit der Objektsysteme zu charakterisieren (,divergence from nature"). Das belege die Hypothese eines Epochenbruchs.

(3) das (Extreme-)Biotechnologie-Verständnis: Gänzlich konträr beziehen sich jene, die die Kontinuität der Synthetischen Biologie belegen wollen, auf Verfahren und Methoden (Kontinuitätsthese). Von einer ,expansion of biotechnology“" spricht Endy (2005, S. 449). Eine ,extreme Gentechnik" sieht die ETC-Gruppe in der Syn- thetischen Biologie (ETC-Group 2007). Denn sie basiere auf etablierten Gen- und Zelltechnologien - wie Nukleotidsynthese, Polymerase Chain Reaction oder Rekombinantes Klonen - und erweitere diese lediglich graduell. Die verwendeten Gensynthese-Technologien gebe es seit den 1970er Jahren. Quantitativ sei man vorangekommen, nicht aber qualitativ.

Jede der drei Verständnisweisen mag an sich mehr oder weniger plausibel sein. ${ }^{2}$ Doch: Sind sie spezifisch genug, um zu erfassen, was den wissenschaftlich-technischen Kern der Synthetischen Biologie kennzeichnet? Und, sind sie allgemein genug, um den zentralen Entwicklungstrend von Zukunftstechnologien zu beschreiben?

Nun findet sich eine weitere, grundlegendere Verständnisweise. Um diese zu erschließen, ist ein anderer Zugang zu wählen - nicht jener über Ziele, Objekte („Ontologie“) oder Verfahren (,Methodologie“), sondern über Prinzipien und Theorien (,Epistemologie“).

\section{Eine vierte Verständnisweise: Nutzung von Selbstorganisationsprinzipien}

Die unterschiedlichen Felder der Synthetischen Biologie haben - über obige drei Verständnisweisen hinaus - ein einigendes Band: das der technischen Nutzbarmachung von Selbstorganisationsprinzipien.

In dieser Verständnisweise spiegelt sich ein allgemeiner Trend wider: „The paradigm of complex, self-organizing systems is stepping ahead at an accelerated pace, both in science and in technology ${ }^{\text {“3 }}$ (Dupuy 2004, S. 12f.). Analog meint die European Technology Assessment Group: ,Sophisticated 'smart' technological systems in the future are expected to have characteristics such as being self-organizing, self-optimizing, self-assembling, self-healing, and cognitive." (ETAG 2009, S. 4) Im Rahmen ihres Minimal-Cell-Mimicry-Projekts heben Luisi und Stano (2011) Ähnliches hervor: „Synthetic cells $[\ldots]$ are relevant for investigating the self-organizing abilities and emergent properties of chemical systems - for example, in originof-life studies and for the realization of chemical autopoietic systems that continuously self-replicate - and can also have biotechnological applications“"(Luisi/Stano 2011). ${ }^{4}$ 
Es seien zudem die Konzepte der Selbstorganisation, die zu einer interdisziplinären Konvergenz von Natur- und Technikwissenschaften beitragen könnten (,technoscience“), so der NSF-Report über Converging Technologies: „Unifying science and engineering“" werde möglich durch „using the concepts of self-organized [...] and complex systems" (Roco/Bainbridge 2002, S. 84). Selbstorganisationskonzepte dienen demnach sowohl der Grundlagen- wie auch der Anwendungsforschung - und weichen die Grenzlinie zwischen beiden auf.

Nimmt man diese programmatischen ÄuBerungen ernst, deutet sich die Entstehung eines neuen Techniktyps an: Sollte er sich durchsetzen, könnte von einer nachmodernen Technik gesprochen werden (Schmidt 2012; Kastenhofer/ Schmidt 2011). Zentrale (zu ,Selbstorganisation" verwandte) Begriffe verweisen auf diesen Wandel: Self-Assembly, Self-Replication und Self-Reproduction, verbunden mit Komplexität, Nichtlinearität, Autopoiesis, Emergenz, Sensitivität, Informationalität, Interaktivität, Flexibilität, Adaptivität sowie Rauschen, Stochastizität, Fluktuationen und gesetzmäßigem Zufall. Von evolutionären Prozessen ist die Rede, von Neuronalen Netzen, Genetischen Algorithmen und Zellulären Automaten.

Mit diesen Begriffen deutet sich ein systemund strukturwissenschaftlich getriebener Wandel im Technikverständnis an, dessen Höhepunkt derzeit die Synthetische Biologie darstellt.

\section{Nachmoderne Technik: Implementierung der System- und Strukturwissenschaften}

Phänomenologisch erscheint nachmoderne Technik nicht als Technik, sondern als (Bio-) Natur: Sie wandelt sich und wächst, sie ist eine ,untechnische" Technik. Sie ist lebend oder sie stellt sich als lebendig dar, sie verfügt über Eigenaktivität und Autonomie. Ihre inneren Dynamiken und Wachstumsphänomene scheinen die Spuren, Signaturen und Siegel des Technischen längst abgestreift zu haben (Karafyllis 2003; Hubig 2006). Nachmoderne Technik hat das Moment der Ruhe und Bewegung offenbar von innen her, nicht von außen.

Wie weit diese „Phäno-Konvergenz“, die Hybridisierung und Amalgamierung von Technik und Natur bereits fortgeschritten ist oder überhaupt fortschreiten kann, ist offen. Treibend - und weit über die Biologie hinausgehend - ist ein weiterer, weitaus grundlegenderer Konvergenztyp, der im gesetzeshaften Kern liegt: die „Nomo-Konvergenz" (nomologische Konvergenz), die auf Strukturgesetzen der Selbstorganisation basiert und einen Systemzugang verfolgt. In unterschiedlichen Objektsystemen finden sich ähnliche Strukturen. „Advanced technologies and biology“, so Csete und Doyle, ,are far more alike in systems-level organization than is widely appreciated" (Csete/Doyle 2002, S. 1664).

Wegbereiter für diese Entwicklung sind die interdisziplinären System- und Strukturwissenschaften ${ }^{5}$, die Strukturen von Objekten unabhängig von ihren jeweiligen materiellen Manifestationen untersuchen. Diese wurden in den 1940er Jahren im Rahmen der Kybernetik und Informationstheorie vorbereitet (erste Welle: Bertalanffy, Wiener, Shannon, von Neumann) und ab den 1960er Jahren in den exakten Naturwissenschaften (zweite Welle: Prigogine, Haken, Maturana/Varela, Foerster) ausformuliert. Prominent sind Dissipative Strukturbildung, Synergetik, Autopoiesis-Konzepte sowie Chaos- und Komplexitätstheorien (Schmidt 2008a). Diese zweite Welle der System- und Strukturwissenschaften ist stark durch die Computerentwicklung befördert worden. In ihrem Zentrum finden sich Selbstorganisationsphänomene.

Die System- und Strukturwissenschaften haben zur Erweiterung der methodischen $\mathrm{Zu}$ gänge beigetragen - auch der der Biologie. Das Forschungsprogramm der Systembiologie, das grundlegend ist für die Synthetische Biologie, ist maßgeblich davon geprägt: „A transition is occurring in biology from the molecular level to the system level that promises to revolutionize our understanding of complex biological regulatory systems and to provide major new opportunities for practical applications" (Kitano 2002, S. 1662).

Mit der technischen Verwendung der System- und Strukturwissenschaften rücken Selbstorganisationsprinzipien ins Zentrum des Diskurses um Zukunftstechnologien. Was sich in der Synthetischen Biologie zeigt, scheint indes nur die Speerspitze eines allgemeinen Trends zu sein: Selbstorganisation spielt eine Rolle (a) in der Robotik, dem Pervasive Computing sowie Software-Agenten, (b) in den Nano- und Mik- 
rosystemtechnologien sowie (c) in den Kognitions-, Neuro- und Pharmakotechnologien.

\section{Prinzipien in der Natur, Versprechungen für die Technik?}

Mit der Natur - das heißt, mit den in der Natur etablierten Prinzipien der Selbstorganisation soll Natur gesteigert und gesteuert, überboten und überwunden werden und somit Technik geschaffen werden. Es ist indes ein spezifischer Naturbegriff: nicht der aus der Physik (Natur als allgemeines Naturgesetz) oder der aus der Lebenswelt, sondern ein Begriff von Natur, der strukturwissenschaftlich ausgerichtet ist - nämlich Natur als jene speziellen Natur(struktur)gesetze, die Selbstorganisation ermöglichen.

Dieser Natur wird viel zugetraut. Sie scheint „besser“ zu sein als der Ingenieur mit seinen Maximen der rationalen Konstruktion (,rational design“"): ${ }^{6}$ Sie verspricht bessere Produkte, Prozesse, Performances.

- Zunächst ist ein Konstruktions- und Kreationsparadigma prominent. Die Nutzung von Selbstorganisationsprozessen dient demnach der Hervorbringung und Herstellung von neuartigen bzw. bislang nur von der Natur erzeugten Produkten: „X wurde in Selbstorganisation hervorgebracht."

- Aber es findet sich auch ein Optimierungsund Effizienzparadigma. Selbstorganisation erscheint als Verfahren und Prozess zur Verbesserung: „Y wurde durch Selbstorganisation optimiert."

- Zudem kann von einem Orientierungsparadigma gesprochen werden. Selbstorganisation kann als Ziel der Entwicklung technischer Produkte verstanden werden, da es die Performance und Verhaltensweise verbessert: „Z ist mit Selbstorganisation(sfähigkeit) ausgestattet.“

Fast erscheint eine selbstorganisationsfähige Technik als handelnd: Sie ist schöpferisch tätig („Produktivität"), wählt zweckrational Mittel (,Optimalität“) und ist mit Entscheidungsfähigkeit ausgestattet (,Adaptivität"). Mitunter wird ihr gar Autonomie und Akteursverhalten zugeschrieben. Was Schelling einst über Natur sagte, gilt heutzutage offenbar auch für Technik: Nachmoderne Technik ist „nicht primitiv“.

\section{Instabilität: Kern der selbstorganisations- basierten Technik}

Die Synthetische Biologie kann, so wurde gesagt, als technische Nutzbarmachung von Selbstorganisationsprinzipien charakterisiert werden. Nun ist der Begriff der Selbstorganisation nicht sonderlich präzise.

Von Kant eingeführt hat er einige Bedeutungsverschiebungen durchgemacht. Doch der semantische Kern bezieht sich seit jeher auf den Prozess der Entstehung von etwas, meistens von etwas Neuem („Emergenz“, Schmidt 2010). Kein Konstrukteur steuert diesen direkt; vielmehr setzt er lediglich Anfangs- und Randbedingungen, weshalb von ,gerichteter" Selbstorganisation gesprochen wird. Der Prozess selbst ist ihm sogar partiell entzogen - entweder aus prinzipiellen (ontologischen) oder aus pragmatischen (methodologischen) Gründen. So umfasst „Selbstorganisation“: Entstehung von etwas, Eigendynamik des Systems sowie Entzogenheit relevanter Details. Von Synthetischen Biologen wird von „Produktivität“, „Prozessualität“ und „Autonomie“" gesprochen.

Die Charakterisierung dessen, was Selbstorganisation kennzeichnet, sagt noch nichts über Quelle und Kern von Selbstorganisation aus: Konstitutiv für Selbstorganisation sind Instabilitäten - das stellen die aktuellen Struktur- und Systemwissenschaften heraus (Schmidt 2008a). „Selbstorganisation wird in der Regel durch eine Instabilität der ,alten“ Struktur gegenüber kleinen Schwankungen eingeleitet", so Ebeling und Feistel (1994, S. 46). Instabilität bezeichnet dabei eine Relation: kleine Ursache, große Wirkung. Sie basiert auf Nichtlinearität, induziert Kipp- und Umschlagpunkte („Bifurkationen“), generiert Rückkopplungs-, Wechselwirkungsund Verstärkungsprozesse, verbunden mit veränderten Kausalitätstypen. Instabilität meint also nicht, dass Systeme prinzipiell kollabieren.

Wer nun, wie die Synthetische Biologie, Selbstorganisation nutzen will, muss Instabilität provozieren: Selbstorganisation bedarf des Durchgangs durch Phasen der Instabilität. Sie ist Bedingung für Symmetriebrüche, die aus lokaler Stabilität und Starre herausführen und so Entwicklung und Evolution, Werden und Wachsen ermöglichen. In Instabilitäten liegt die techno-ontologische Quelle jener Aktivität und Produktivität, auf die die Syn- 
thetische Biologie abzielt. Instabilitäten erfahren somit eine Positivierung - wie auch Rauschen und stochastische Fluktuationen.

\section{Grenzen von Konstruktion und Kontrolle}

Allerdings sind Instabilitäten zweischneidig. Denn sie sind nicht nur Quelle der Produktivität. Vielmehr führen sie auch zu Grenzen von Konstruktion und Kontrolle. Diese Beschränkungen werden ebenfalls von den System- und Strukturwissenschaften nahegelegt. Sie zeigen, warum es so schwer ist, biologische Systeme als technische Objekte zu begreifen und zu behandeln.

Sieht man also Instabilitäten als Kern einer selbstorganisationsfähigen Technik an, werden Grenzen eines rationalen Designs deutlich: Grenzen der Prognostizierbarkeit und (Re-) Produzierbarkeit treten auf. Denn bei Instabilität kommt es auf allerkleinste Details an: kleine Ursache, große Wirkung. Technisch können diese Details - d. h. Ursachen, manifest in Anfangs- und Randbedingungen - aus prinzipiellen wie aus pragmatischen Gründen nicht beherrscht werden (Schmidt 2008b). Das gilt einerseits für den technischen Zugriff auf lebende Systeme (,top-down“; Intervention, Manipulation) und andererseits für die technische Herstellung und Hervorbringung lebender Systeme (,bottomup"; Kreation): Je weiter der technische Zugriff auf Natur voranschreitet, desto deutlicher tritt das Unverfügbare und Unbestimmte, das Nichtkontrollierbare und Nichtwissen hervor.

Rückblickend betrachtet ist es also durchaus verständlich, weshalb Instabilitäten in der klassisch-modernen Technik nichts verloren hatten. Wo sie auftraten, waren sie störend; es galt, sie zu eliminieren: Technik war Technik, insofern sie stabil war. Nur bei Stabilität sind die Bedingungen von Konstruier- und Kontrollierbarkeit erfüllbar.

\section{8 „Kollaborative Technik“}

Es war der Philosoph und Ethiker des Prinzips Verantwortung, Hans Jonas, der schon in den 1980er Jahren ahnte, welche Problemlage mit einer im Entstehen begriffenen selbstorganisationsfähigen Technik verbunden sein könnte.

Jonas hat die Differenz zwischen „Ingenieurkunst" (klassisch-moderne Technik) und „organischer" bzw. „biologischer" Technologie (Teil der nachmodernen Technik) in den Blick genommen (Jonas 1987, S. 163ff.). ${ }^{7}$ „Bei totem Stoff“, so Jonas über die bisherige (klassischmoderne) Technik, ,,ist der Hersteller der allein Handelnde gegenüber dem passiven Material. Bei Organismen [hingegen] trifft Tätigkeit auf Tätigkeit: biologische Technik ist kollaborativ mit der Selbsttätigkeit eines aktiven ,Materials““ (ders., S. 165).

Äußerst weitsichtig hat Jonas einige Charakteristika dieser neuen Technik zusammengestellt (ders., S. 164ff.): (a) Selbsttätigkeit und Kollaborativität, (b) Komplexität, Dynamik und mangelnde Prognostizierbarkeit, (c) Individualität und Beschränkung der (technischen) Kontrollier- und Reproduzierbarkeit, (d) Unumkehrbarkeit, Irreversibilität und Historizität. Diese neue Technik - so Jonas weiter - verändere das technische Handeln des Ingenieurs und seine ,,mittelbare Kausalbeziehung“ zum technischen Gegenstand: ,"Herstellen“ heißt hier Entlassen in die Strömung des Werdens, worin auch der Hersteller treibt" (ders., S. 168).

Auch wenn Jonas die Grenze zwischen „organischer“ und „Ingenieurstechnik“ allzu schematisch gezogen haben mag, so bleibt doch eine graduelle Gegenüberstellung der beiden Techniktypen. Was Jonas allerdings entgeht, ist, dass seine Differenzierung viel grundlegender ist, als er sie darstellt: Nicht das Organische, sondern die Selbstorganisationsfähigkeit mit ihren Instabilitäten ist entscheidend. Was von Instabilitäten bestimmt wird, wie beispielsweise insbesondere das Organische, entzieht sich einer weitgehenden Konstruktions- und Kontrolllogik. Es bleibt eine Domäne des Unverfügbaren und Unbestimmbaren.

Dass bei der Synthetischen Biologie dennoch von „Technik“ die Rede ist, ist bemerkenswert. Denn es ist eine Spannung zwischen einer selbstorganisationsfähigen (nachmodernen) „Technik“ einerseits und dem Anspruch auf rationale Konstruktion und Kontrolle andererseits zu konstatieren. Letzteres wurde von der berühmten Expertengruppe der EU unmissverständlich als Anspruch formuliert: „In essence, synthetic biology will enable the design of 'biological systems' in a rational and systematic way." (EC 2005, S. 5) Angesichts der Instabilitäten wird man Zweifel artikulieren müssen, ob dieser Anspruch nicht fehlgeht. 


\section{Fazit: Herausforderung für die TA}

Instabile Systeme, wie sie in der Synthetischen Biologie auftreten, können in ganz anderer Weise außer Kontrolle geraten als klassisch-moderne Technik. Metaphorisch gesprochen: Wer Instabilität provoziert, tanzt auf des Messers Schneide! Tucker und Zilinskas haben die „Promise and Perils of Synthetic Biologie“ untersucht: „Because engineered micro-organisms are self-replicating and capable of evolution, they belong in a different risk category than toxic chemicals or radioactive materials." (Tucker/Zilinskas 2006)

Das gilt unzweifelhaft schon für die herkömmliche Biotechnologie und steigert sich nochmals in der Synthetischen Biologie. Risikoabschätzungen - als Teil der TA - sind im Feld der Synthetischen Biologie schwerlich möglich. Denn sie beruhen auf dem ,Ähnlichkeitsprinzip“: Wenn ein neues Biosystem einem bekannten ähnlich ist, werde es sich ähnlich verhalten; die Risiken werden als ähnlich veranschlagt. Doch von Ähnlichkeit kann keine Rede sein, wo sich Biosysteme aus sich heraus selbst konstruieren. Hier gibt es keine Vergleichbarkeit, auf die man sich in Analyse und Bewertung stützen könnte. Das Ähnlichkeitsprinzip scheitert also am „ontologischen Kern" der nachmodernen Technik und an der von ihr erzeugten Komplexität. Nichtwissen und Unsicherheit werden von der Technik selbst - nicht erst im gesellschaftlichen Verwendungskontext produziert. „The novel kind of uncertainty that is brought about by those new technologies is intimately linked with their being able to set off complex phenomena." (Dupuy 2004, S. 10)

Was bedeutet die qualitative, durch den Kern der Technik gesteigerte Komplexitäts- und Unbestimmbarkeitsproblematik für die TA? Gewiss, TA ist nicht am Ende. Doch TA sollte kritischer als bisher den derzeitigen Trend zur nachmodernen Technik antizipieren, um techniknahe Gestaltungsoptionen von Wissenschaftsund Forschungskorridoren auszuloten. Folgende Fragen wären verstärkt zu stellen: Wie kann die Forschung von eigensicheren Biosystemen in der Synthetischen Biologie gestärkt werden? Wodurch sind solche Systeme definiert und welchen Kriterien müssen sie genügen? Bei welchen Techniklinien der Synthetischen Biologie ist ein Nachhaltigkeitspotenzial zu erwarten?
Grundlegender noch erscheint allerdings die Frage, ob und wie stark unsere spätmodernen Wissenschaftsgesellschaften auf eine instabilitätsbasierte selbstorganisationsfähige Technik setzen sollten? Schließlich wird mit dieser stetig Nichtwissen und Nichtkontrollierbarkeit produziert. Hier wäre die Maxime der Zurückhaltung und „Heuristik der Furcht", verbunden mit bewahrenden Vorsorge- und Vorsichtsprinzipien angebracht. Hans Jonas antizipierte diese Herausforderungen in unübertroffener Klarheit.

Bleibt also wenig „Hoffnung“? Trotz aller prinzipiellen Zurückhaltung wäre nach möglichen Seitenarmen und spezifischen Pfaden der Synthetischen Biologie Ausschau zu halten, die dazu beitragen könnten, was Ernst Bloch als „Allianztechnik" und „Mitproduktivität der Natur" bezeichnet hat (Bloch 1959, S. 802). Derartiges wird mit der herkömmlichen klassisch-modernen Technik nicht zu haben sein. Denn diese liefert ,nicht einmal eine Treppe, geschweige eine Tür zur möglichen Naturallianz" (ders., S. 813). Innerhalb der vielen Facetten der nachmodernen Technik könnte es Felder geben, in denen die Dinge anders liegen - und sich Potenziale für die Entwicklung einer an Nachhaltigkeitskriterien orientierten Allianztechnik finden lassen. Synthetische Biologie könnte sodann durchaus zu einer nachhaltigen Technikentwicklung beitragen. $\mathrm{Ob}$ das möglich ist, ist bis dato unbestimmt und unbestimmbar.

\section{Anmerkungen}

1) Der Autor dankt dem BMBF für einen Unterauftrag im Rahmen des von A. v. Gleich (Universität Bremen, Fachgebiet Technik Gestaltung) geleiteten BMBF-Projekts ,SynBioTA. Innovations- und Technikanalyse Synthetische Biologie“. Dank gilt B. Giese und A. v. Gleich (Bremen) sowie A. Nordmann (Darmstadt) für weiterführende Diskussionen.

2) Sie mögen sich partiell ergänzen, verbleiben alle im Horizont der Biologie (als Disziplin).

3) Siehe auch VDI 2003, S. 5.

4) Der VDI hat vor einiger Zeit bereits eine Studie zu „Ansätze[n] zur technischen Nutzung der Selbstorganisation“ vorgelegt (VDI 2003).

5) Siehe dazu Weizsäcker 1974, S. 22f.

6) Mit der Zuschreibung technisch-funktionaler Kategorien zur Natur sind metaphysische, handlungstheoretische und anthropomorphe Unterstellungen verbunden.

7) Vgl. auch Köchy 2010. 


\section{Literatur}

Bloch, E., 1959: Das Prinzip Hoffnung. Frankfurt Csete, M.E.; Doyle, J.C., 2002: Reverse Engineering of Biological Complexity. In: Science 295/5560 (2002), S. 1664-1669

DFG - Deutsche Forschungsgemeinschaft, acatech - Deutsche Akademie der Technikwissenschaften; Deutsche Akademie der Naturforscher Leopoldina, 2009: Synthetische Biologie. Bonn

Dupuy, J.P., 2004: Complexity and Uncertainty a Prudential Approach to Nanotechnology. European Commission; http://www.europa.eu.int/comm/health/ ph_risk/documents/ev_20040301_en.pdf (download 12.10.12)

Ebeling, W.; Feistel, R., 1994: Chaos und Kosmos. Prinzipien der Evolution. Heidelberg

EC - European Commission, 2005: Synthetic Biology. Applying Engineering to Biology. Report of a NEST High-Level Expert Group EU 21796. Brüssel

Endy, D., 2005: Foundations for Engineering Biology. In: Nature 438 (2005), S. 449-453

ETAG - European Technology Assessment Group, 2009: Making a Perfect Life. Bioengineering in the 21st century. Den Haag

ETC-Group - Action Group on Erosion, Technology and Concentration, 2007: Extreme Genetic Engineering. An Introduction to Synthetic Biology; http://www. etcgroup.org/sites/www.etcgroup.org/files/publication/602/01/synbioreportweb.pdf (download 12.10.12) Hubig, C., 2006: Die Kunst des Möglichen I. Bielefeld Jonas, H., 1987: Laßt uns einen Menschen klonieren. In: Jonas, H.: Technik, Medizin und Ethik. Frankfurt Karafyllis, N.C. (Hg.), 2003: Biofakte. Paderborn

Kastenhofer, K.; Schmidt, J.C., 2011: On Intervention, Construction and Creation: Power and Knowledge in Technoscience and Late-modern Technology. In: Zülsdorf, T.B.; Coenen, Chr.; Ferrari, A. (Hg.): Quantum Engagements: Social Reflections of Nanoscience and Emerging Technologies. Heidelberg

Kitano, H., 2002: Looking Beyond the Details: A Rise in System-oriented Approaches in Genetics and Molecular Biology. In: Current Genetics 41/1 (2002), S. 1-10 Köchy, K., 2010: Sind die Überlegungen von Hans Jonas zum Sonderstatus biologischer Technik angesichts der Entwicklung in der Synthetischen Biologie noch haltbar? Universität Kassel, unveröffentlichtes Manuskript

Liebert, W.; Schmidt, J.C., 2010: Towards a Prospective Technology Assessment: Challenges and Requirements for Technology Assessment in the Age of Technoscience. In: Poiesis \& Praxis 7/1-2 (2010), S. 99-116
Luisi, P.L.; Stano, P., 2011: Synthetic Biology: Minimal Cell Mimicry. In: Nature Chemistry 3 (2011), S. 755-756

Pühler, A.; Müller-Röber, B.; Weitze, M.-D. (Hg.), 2011: Synthetische Biologie. Die Geburt einer neuen Technikwissenschaft. Berlin

Roco, M.C.; Bainbridge, W.S. (Hg.), 2002: Converging Technologies for Improving Human Performance. Arlington

Schmidt, J.C., 2008a: Instabilität in Natur und Wissenschaft. Berlin

Schmidt, J.C., 2008b: Unbestimmtheit der Nanoforschung. Über Kontrolle der (und in der) Nanotechnoscience. In: Köchy, K.; Norwig, M.; Hofmeister, G. (Hg.): Nanobiotechnologien. Philosophische, anthropologische und ethische Fragen. Freiburg, S. 37-54

Schmidt, J.C., 2010: Emergence and Emergent Properties in Nanotechnology. In: Guston, D. (Hg.): Encyclopedia of Nanoscience and Society. New York, S. 180-184

Schmidt, J.C., 2012: Quellen des Nichtwissens. Ein Beitrag zur Wissenschafts- und Technikphilosophie des Nichtwissens. In: Janich, N.; Nordmann, A.; Schebek, L. (Hg.): Nichtwissenskommunikation in den Wissenschaften. Frankfurt, S. 93-124

TESSY-Consortium, 2008: Information Leaflet: Synthetic Biology in Europe; http://www.tessy-europe. eu/public_docs/SyntheticBiology_TESSY-Information-Leaflet.pdf (download 12.10.12)

Tucker, J.B.; Zilinskas, R.A., 2006: The Promise and Perils of Synthetic Biology. In: The New Atlantis 12 (2006), S. 25-45

VDI - Verein Deutscher Ingenieure (Hg.), 2003: Ansätze zur technischen Nutzung der Selbstorganisation. Düsseldorf

Weizsäcker, C.F. v., 1974: Die Einheit der Natur. München

\section{Kontakt}

Prof. Dr. Jan C. Schmidt

h_da

Darmstadt University of Applied Sciences

Department of Social Sciences

Unit of Social, Culture and Technology Studies

Haardtring 100, 64295 Darmstadt

Tel.: + 49 (0) 6151 - 168742

E-Mail: jan.schmidt@h-da.de 